Medien zu selbst gesetzten Zwecken, aber auch das Hintergrundwissen über Medien (zum Beispiel rechtliche Fragen)“ (S. 305f) bei Jugendlichen wenig entwickelt waren. Daher fordert der Autor (erneut), medienerzieherische Inhalte in Schule und Jugendarbeit verstärkt zu vermitteln, insbesondere die inzwischen dominierende „informationstechnische Bildung“ mit der „Medienerziehung“ $\mathrm{zu}$ verbinden (wie es die Kultusministerkonferenz schon in den 90er Jahren gefordert hat) und eine so breit verstandene Medienbildung zur Querschnittsaufgabe und zum übergreifenden Ziel pädagogischer Bemühungen zu machen. Dass es mit diesen Forderungen selbst in Bayern nicht sehr weit her ist, muss der Autor am Ende einräumen. So bleibt das Motto eines der vielen Pilot- und Vorzeigeprojekte „Mediageneration - kompetent in die Medienzukunft" bis dato ein beschämendes Desiderat.

Hans-Dieter Kübler

\section{Steffen Kolb}

\section{Mediale Thematisierung in Zyklen}

Theoretischer Entwurf und empirische Anwendung

Köln: Herbert von Halem, 2005. - 334 S.

ISBN: 3-938258-05-0

Das Buch, die Dissertation des Autors, besteht aus zwei Hauptteilen: In einem Theorieteil werden bestehende Ansätze zur Beschreibung/ Erklärung von medialen Themenverläufen dargestellt, diskutiert und zu einem idealtypischen "Zyklusmodell“ des Themenverlaufs verdichtet. In einem empirischen Teil wird als Fallstudie die Karriere des Themas „Blei im Benzin“ (bzw. allgemeiner: „umwelt- und gesundheitsschädliche Wirkungen von Fahrzeugabgasen“) von 1965-2000 nachgezeichnet. Man kann das Buch also aus zwei Perspektiven lesen: Als Darstellung der Karriere eines Umweltthemas oder als Versuch der Theoriebildung über die Dynamik medialer Thematisierung. Der Titel des Buches bezieht sich auf die zweite Perspektive. D. h. der Autor möchte seine Dissertation in erster Linie als Beitrag zur Entwicklung einer Theorie medialer Thematisierung verstanden wissen und die Darstellung des Umweltthemas als erste empirische Überprüfung des „Themenzykluskonzepts“ (S. 125). Leider wird das Buch diesem theoretischen Anspruch nur bedingt gerecht. Die Karriere des Themas „Umwelt-/Gesundheitsbelastungen von Autoabgasen" differenziert nachzuzeichnen, gelingt dem Autor dagegen recht gut. Ich beginne meine Besprechung mit dem relativ unproblematischen zweiten Teil, also mit der inhaltsanalytischen Fallstudie, um danach ausführlicher auf die theoretischen Überlegungen zur medialen Thematisierung und die Frage ihrer empirischen Validierung einzugehen.

Gegenstand der Fallstudie ist die Berichterstattung in deutschen, britischen und französischen Printmedien über Umweltprobleme durch Autoabgase, wobei der Schwerpunkt der Analyse und Darstellung auf der deutschen Berichterstattung liegt (FAZ, SZ, Bild, Spiegel). Der Autor zeichnet die mediale Darstellung des Themas von 1965 bis 2000 differenziert nach und zeigt sich dabei sowohl bei der Darstellung zeitlicher Trends als auch beim internationalen Vergleich methodisch sehr reflektiert.

Die Analyse zeigt die Bedeutung der Wissenschaft als Themenprotagonist in den Anfangsphasen des Themas und die Politisierung des Themas in der Phase hoher Intensität, die sich beispielsweise im Anstieg des Anteils der in der Berichterstattung genannten politischen Akteure und im Rückgang des Anteils von Akteuren aus dem wissenschaftlich-technischen System ausdrückt. Auch die Berichterstattungsanlässe kamen in den "heißen Phasen“ überdurchschnittlich häufig aus dem politischen System, und es wurde besonders häufig auf politische Prozesse (z. B. Gesetzgebungsverfahren) Bezug genommen. Interessant ist auch, dass während der Phase der intensiven politischen Befassung mit dem Thema der Anteil der Artikel sinkt, in denen auf Umweltschäden verwiesen wird. Dies lässt sich mit der politischen Eigendynamik erklären und der Tatsache, dass der umweltpolitische Problembezug zugunsten der politischen Logik (Durchsetzung einer neuen Regulation) zurücktritt bzw. implizit als gegeben vorausgesetzt wird. Schließlich ändert sich das Framing des Themas: Während in den Anfangs- und Endphasen vor allem der wissenschaftliche, technische und ökologische Kontext relevant ist, dominiert in den besonders intensiven mittleren Themenphasen die politische Kontextualisierung. In der letzten Phase, in der es vermutlich besonders um die Implementierung der neuen Regulation geht (Umstellung auf bleifreies Benzin, Umrüstung auf Katalysator), gewinnt auch die verbrau- 
cher- und serviceorientierte Perspektive an Bedeutung.

Insgesamt zeichnet der Autor das Bild einer erfolgreichen Problemlösung durch öffentliche Thematisierung. Nach wissenschaftlichen Warnungen vor den Gesundheitsgefahren von Blei bzw. von Umweltrisiken der Autoabgase insgesamt, die mediale Aufmerksamkeit finden, nimmt sich die Politik des Problems an. Es entwickelt sich ein gesellschaftlicher Problemlösungsprozess, in dessen Verlauf nach amerikanischem und japanischem Vorbild der Abgaskatalysator eingeführt und als notwendige Vorbedingung die Treibstoffversorgung auf bleifreies Benzin umgestellt wird. Während dieses Prozesses fungieren die Medien zunächst im Sinne des Agenda Setting, dann als Diskursarena, während sie in der abschließenden Implementierungsphase auch Ratgeberfunktion für die Bürger wahrnehmen.

Trotz der vom Autor eingeräumten methodischen Probleme des Zugangs zu der relevanten Berichterstattung in Großbritannien und Frankreich ist auch der internationale Vergleich recht aufschlussreich, weil er auf die Kontingenz der Themenverläufe verweist. Ein Thema kann sich so entwickeln wie im deutschen Fall, aber eben auch anders. In Frankreich setzte die Thematisierung später ein als in Deutschland (vermutlich durch die deutsche Initiative angestoßen) und Experten spielten als Themenprotagonisten keine Rolle, wurden dafür aber - anders in Deutschland - in den späteren Phasen sehr relevant. Auch der Verweis auf ökologische Risiken fehlte, anders als in Deutschland, in den ersten Phasen, gewann in den späteren Phasen dagegen an Bedeutung. Der dominante Themenkontext hat sich offenbar von einem industrie- und handelspolitischen $\mathrm{zu}$ einem ökologischen Framing gewandelt.

Der grobe Verlauf der britischen Thematisierung ähnelt dem deutschen, allerdings mit einer zeitlichen Verschiebung und semantisch offenbar mit einer eindeutigeren Fokussierung auf die ökologischen Probleme von Blei (statt von Autoabgasen insgesamt). Experten spielten in der britischen Debatte keine so bedeutende Rolle wie in Deutschland und Frankreich; allerdings sind Akteure aus der Wirtschaft ungewöhnlich oft in der Berichterstattung vertreten - häufiger sogar als politische Akteure. (Vielleicht ist das jedoch ein Artefakt, weil sich der Autor bei der Materialbeschaffung stark auf Archive von Industrieverbänden stützte, im britischen Fall auf das Archiv des „Institute of Petroleum“.)

Kommen wir nun $\mathrm{zu}$ den theoretischen Überlegungen des Autors. Implizit geht der Autor von der von mir geteilten Annahme aus, dass es eine Eigendynamik des öffentlichen (medialen) Diskurses gibt, in der es zur Herausbildung von Sinnkomplexen kommt, die gesellschaftliche Aufmerksamkeit lenken und offene Bindungsstellen für anschlussfähige Kommunikation (für gesellschaftliche Akteure und Journalisten) schaffen. Der Autor stützt sich bei seinen Überlegungen auf eine Reihe von Ansätzen: die Thematisierungskonzepte u. a. von Niklas Luhmann und Anthony Downs, kommunikationswissenschaftliche Theorien zum Agenda Setting und zur Nachrichtenselektion (Nachrichtenwerte) sowie das in verschiedenen Disziplinen (u. a. der wirtschaftswissenschaftlichen Innovationsforschung) verwendete mathematisch begründete Diffusionsmodell. Aus diesen Zutaten hätte man in der Tat etwas machen können, doch dem Autor fehlt das richtige Rezept. Er belässt es weitgehend bei der Konzeptionierung eines eigenen Phasenmodells, das jedoch nicht systematisch aus Prämissen abgeleitet, sondern mit mehr oder weniger schlüssigen Plausibilitätsüberlegungen begründet wird, die nicht über die heuristischen Modelle von Luhmann und Downs hinausgehen. Den Einbezug formaler Überlegungen zur Abgrenzung der Phasen aus der angeblichen Normalverteilungsform der (idealisierten) Themen halte ich sogar für unberechtigt, weil die Normalverteilung auf einem mathematischen Modell der Diffusion beruht, das in dieser Form für die Themendynamik sicherlich nicht gültig ist (siehe unten).

Der Autor diskutiert im Theorieteil eine Reihe wirklich wichtiger Fragen, etwa nach der Definition eines Themas, nach der Typologie von Themen mit u. U. verschiedenen Verläufen und nach dem Vorliegen einer Themenhierarchie. Zumindest die beiden ersten Fragen werden aber nicht wirklich beantwortet. Die Frage der Definition eines Themas ist aber ziemlich entscheidend: Ist ein Thema ein analytisches Konstrukt, das sozusagen beliebig durch Selektionsregeln definiert werden kann, oder ist es im Sinne Luhmanns ein öffentlicher "Sinnkomplex“, für den jeweils ein semantischer Existenznachweis zu führen ist, d. h. für den zu zeigen ist, dass er von Kommunikationsteilnehmern tatsächlich zur Konstruktion 
eines Anschlusses für die Kommunikation verwendet wird?

Im Hinblick auf die Typologie von Themen fehlt eine Eingrenzung des Geltungsbereichs der vom Autor entwickelten theoretischen Vorstellungen. Sowohl Luhmann als auch Downs unterstellen implizit eine bestimmte Thematisierungsform: Die Entstehung/Entdeckung eines neuen Problems wird durch öffentliche Thematisierung in die politische Problembearbeitung überführt und ggf. gelöst. Diese Art von Themen scheint auch der Autor im Blick $\mathrm{zu}$ haben. Es sind aber keineswegs die einzig vorkommenden. Für welche Art von Themen gilt also der vorgestellte Themenzyklus? Aus Japan ist beispielsweise bekannt (Hayashi 2006), dass die Thematisierung von Erdbeben einem Sägezahnmuster folgt: Nach einem Erdbeben steigt die Zahl der Berichte schlagartig, um dann - je nach Schwere - im Verlauf von Wochen oder Monaten exponentiell abzufallen. $\mathrm{Zu}$ den Jahrestagen gibt es dann jeweils noch kurzzeitige schwächere Maxima in der Intensitätsverteilung. Zum Leidwesen der japanischen Katastrophenvorsorge wird das Thema „Erdbeben" zwischen den einzelnen Ereignissen kaum behandelt. Ähnliche Verläufe dürfte es auch in Deutschland bei Themen geben, die stark von Ereignissen in der Systemumwelt getriggert sind, ohne jedoch selbstreferentielle politische Prozesse auszulösen.

Schwerwiegender als die nicht überzeugende Herleitung des 5-phasigen Themenzyklus-Modells ist, dass entgegen der erklärten Absicht des Autors die Entwicklung einer Theorie nicht ernsthaft in Angriff genommen wird. Eine Theorie der medialen Thematisierung müsste $\mathrm{m}$. E. die zeitliche Dynamik der Thematisierung, also ihre Intensität, vielleicht auch semantische Merkmale (z. B. Politisierungsgrad), auf die zugrunde liegenden Prozesse zurückführen. Als Beispiel mag das vom Autor selbst angeführte Infektionsmodell der Diffusionsforschung dienen. Hier ergibt sich die beobachtete Form der Kurve - Normalverteilung für die Neuinfektionen bzw. S-Kurve für den Anteil der bereits „infizierten“ Population - mathematisch aus zwei angenommenen Einzelprozessen, einer fördernd, der andere hemmend: (1) die Wahrscheinlichkeit der Infektion eines bislang nicht infizierten Individuums steigt mit steigendem Anteil der bereits infizierten Population und (2) die Rate der Neuinfektionen sinkt mit dem Anteil der bereits infizierten Population, weil sich Individuen innerhalb des beobachteten Zeitraums nicht zweimal infizieren können (Sättigung).

Dieses Infektionsmodell könnte tatsächlich heuristischer Ausgangspunkt für die Entwicklung einer Theorie medialer Thematisierung sein, allerdings nicht durch die unkritische Übernahme der von diesem Modell postulierten "Normalverteilung" in die Theorie der Thematisierung, wie es der Autor tut. Das Diffusionsmodell zeigt, dass es auf die Kombination fördernder und hemmender Prozesse ankommt, die die Form der Thematisierungskurve bestimmen. Im Falle der medialen Thematisierung werden diese Prozesse sicherlich wesentlich komplexer und kontingenter als im Falle des einfachen Diffusionsmodells sein. Die fallspezifische Unterscheidung solcher Faktoren könnte dann auch Anlass für eine theoretisch fundierte Typologie von Thematisierungsformen sein.

Kommunikationswissenschaftlich interessant wären vor allem solche Faktoren, die auf eine mediale Eigendynamik (z. B. auf mediale Selbstreferenzen) hinweisen. Interessanterweise gilt „Thematisierung“ bei Schulz (1976) als einer der Nachrichtenfaktoren, d. h. es gibt empirische Evidenz für einen selbstreferentiellen, verstärkenden Prozess, der - analog zur Erhöhung der individuellen Infektionswahrscheinlichkeit mit Fortschreiten der Infektion in der Population - die Orientierung von Selektionsentscheidungen an den durch vergangene Selektionsentscheidungen entstandenen Sinnkomplexen impliziert. D. h. Journalisten wählen ceteris paribus bevorzugt solche Artikel aus, die sich einem bereits etablierten Thema zuordnen lassen. Der Autor verweist zwar (zu Recht) auf die Nachrichtenwerte als ein Konzept mit Relevanz für die Themendynamik, erwähnt auch den Faktor „Thematisierung“, unterlässt aber die auf der Hand liegende Diskussion dieses besonders einschlägigen Nachrichtenfaktors. Ein zweiter „fördernder“ Prozess ist der politische Agenda Setting-Effekt. Durch ihn nehmen die themenbezogenen Aktivitäten von gesellschaftlichen Akteuren zu, so dass der entsprechende Informationsanfall bei den Primärkommunikatoren steigt. Auch hier nennt der Autor zwar Agenda Setting als allgemein relevanten Effekt, diskutiert aber diesen Effekt nicht explizit als fördernden Prozess für Themenbildung.

Schwieriger ist es, funktionale Äquivalente 
für den zweiten Faktor des Diffiusionsmodells - Sättigung - zu finden. In der Diffusionsforschung geht man davon aus, dass ein einmal Infizierter innerhalb der betrachteten Zeitperiode nicht ein zweites Mal infiziert werden kann. Dies ist im Falle des Journalismus aber gerade nicht der Fall. Journalisten können und werden in der Regel häufiger Artikel zum gleichen Thema veröffentlichen. Eine theoretische Sättigung nach dem Modell der erschöpften Population wäre erst gegeben, wenn sich alle Artikel mit nur einem Thema befassen würden.

Tatsächlich bleiben aber selbst „heiße“ Themen weit unterhalb dieser theoretischen Grenze und empirisch zeigt sich, das die Intensität von Themen auch wieder abnimmt. Es ist also klar, dass es hemmende Effekte gibt, sonst würde sich aufgrund der Wirkung des ersten Faktors ein Thema solange ausbreiten, bis nur noch Artikel zu diesem Thema veröffentlicht würden. Solche hemmenden Effekte könnten z. B. die Themenkonkurrenz sein oder Relevanzänderungen beim Publikum und/oder den gesellschaftlichen Akteuren, z. B. weil eine akzeptable Problemlösung gefunden wurde oder das Problem als unlösbar gilt und zunächst verschoben wird. Eine Theorie medialer Thematisierung müsste empirisch überprüfbare Hypothesen über solche Prozesse enthalten.

Neben den angedeuteten Faktoren, die die Eigendynamik medialer Themenkarrieren bestimmen, gibt es externe Faktoren, die in den idealisierten eigendynamischen Prozess eingreifen. $\mathrm{Zu}$ diesen Faktoren rechne ich z. B. Akteurstrategien (z. B. Issue Management) und systemexterne Ereignisse (z. B. Katastrophen).

Abschließend möchte ich noch auf die empirische Überprüfung des Themenzyklus-Modells durch die empirische Fallstudie eingehen. Absehen möchte ich von methodischen Einwänden (Konstanz der Selektionskriterien über die Zeit und zwischen den drei Ländern, teilweise geringe Häufigkeiten, nur ein Fallbeispiel für die Validierung einer Theorie mit Allgemeingültigkeitsanspruch), die der Autor selbst diskutiert und die sicher teilweise auf die beschränkten Möglichkeiten im Rahmen einer Dissertation zurückzuführen sind.

Für mich stellt sich aber die grundsätzlichere Frage, ob der Fallstudie tatsächlich ein „Thema“ im Sinne einer einheitlichen Sinnstruktur zugrunde liegt. Für die Inhaltsanalyse ausgewählt wurden nach Angaben des Autors (S. 133ff.) Artikel, in denen es direkt oder indirekt um die Frage des Bleigehalts im Benzin ging. Dazu gehört eine frühe Auseinandersetzung mit den Gesundheitsgefahren durch Blei im Benzin (in der ersten Hälfte der 70er Jahre) sowie die intensivere und länger andauernde Auseinandersetzung mit der Abgasentgiftung durch den Katalysator Mitte der 80er Jahre, bei der zwar die Gesundheits- und Umweltgefährdung durch Autoabgase allgemein eine wichtige Rolle spielten, das Blei im Benzin dagegen in erster Linie als "Gefahr" für den Katalysator und der Verzicht auf Blei im Benzin als „Risiko“ für den Automotor behandelt wurde. Entsprechend war die Einführung des bleifreien Benzins nicht in erster Linie durch Gesundheitsbedenken motiviert, sondern technische Voraussetzung für die Einführung des Autokatalysators.

Ich gehe auf die Frage der Auswahlkriterien für die analysierten Artikel so ausführlich ein, weil diese quasi die operationale Themendefinition darstellen. Der Autor behandelt beide Diskussionsstränge - Reduzierung des Bleigehalts im Benzin Anfang der 70er Jahre und Einführung des bleifreien Benzins im Zuge der Katalysatoreinführung - als Teilthemen eines Themas auf der Mesoebene und interpretiert nach seinem Phasenmodell das erste Einzelthema als Phase der „Schlüsselereignisse“ im Kontext des Mesothemas. Mir erscheint es jedoch relativ unwahrscheinlich, dass die Diskussion um die Bleireduzierung Anfang der 70er Jahre als Schlüsselereignis die spätere Diskussion um die Gefährlichkeit von Autoabgasen allgemein und die Einführung des Abgaskatalysators stimuliert hat, weil die Bleireduzierung im Benzin nicht den Katalysator erforderte, sondern umgekehrt bleifreies Benzin eine technische Voraussetzung für die Einführung des Katalysators darstellte. Die Katalysatoreinführung ist also nicht die Lösung für ein durch Schlüsselereignisse um die Gesundheitsgefährdung durch Blei aufgeworfenes neues Problem. Dass sich die spätere öffentliche Diskussion teilweise auch wieder mit „Blei im Benzin“ befasste, ist m. E. also eher ein Zufall. Letztlich zu beantworten wäre diese Frage, ob es sich um ein Thema oder mehrere Themen handelt, nur durch eine eingehende semantische Analyse des Medieninhalts, in der geklärt werden müsste, ob die spätere allgemeinere Debatte über die Gefährlichkeit von Autoabgasen semantisch an die vorangegangene Debatte anschloss oder nicht. Da es diese Analyse nicht gibt, ist es frag- 
lich, ob inhaltliche Unterschiede in der Struktur der Berichterstattung zwischen den Phasen tatsächlich als semantische Verschiebung im Themenverlauf oder nicht teilweise eher als Übergang von einem zu einem anderen Thema interpretiert werden müsste.

Die Anwendung des 5-phasigen Themenzykluskonzepts auf die inhaltsanalytische Fallstudie ist hochgradig interpretationsbedürftig, um nicht zu sagen in weiten Grenzen willkürlich. Zudem entspricht die Fallstudie nicht dem theoretischen Modell - so findet der Autor empirisch sechs statt der von seinem Modell geforderten fünf Phasen. Ist damit die "Theorie“ falsifiziert? Nicht für den Autor. Er schreibt: „Allerdings zeigt sich schon in der ersten empirischen Anwendung, dass ein Themenzykluskonzept flexibel sein muss." (S. 182) Im Klartext heißt das, dass die vorkommenden Thematisierungsverläufe empirisch eine große Varianz aufweisen, die theoretisch nicht verstanden werden. Eine Theorie müsste zudem gehaltvoll sein, d. h. zum Beispiel Aussagen über inhaltliche Charakteristika der verschiedenen Phasen machen. Inhaltliche Unterschiede in der Berichterstattung in den verschiedenen Phasen findet der Autor zwar, doch werden diese nicht theoretisch postuliert und folgen in den drei Ländern auch nicht dem gleichen Muster.

Nun muss man konstatieren, dass der Autor selbst an vielen Stellen die Grenzen seiner theoretischen Überlegungen und ihrer empirischen Überprüfung einräumt, sich der Vorläufigkeit und des spekulativen Charakters seiner Analyse also bewusst ist. Trotzdem muss man ihn an seinem eigenen theoretischen Anspruch messen, der durch den Titel des Buches erhoben wird. Die Bausteine einer möglichen Theorie der medialen Thematisierung werden in dem Buch durchaus bereit gestellt und insofern ist es auch mit Gewinn zu lesen. Leider werden diese Bausteine dann aber nicht zu einem tragfähigen Gebäude zusammengesetzt. Letztlich erbringt der Theorieteil des Buches deshalb nicht wesentlich mehr an theoretischen Einsichten in den Prozess medialer Thematisierung, als Niklas Luhmann bereits 1970 in wenigen Sätzen in seinem bekannten Aufsatz über „Öffentliche Meinung" skizziert hatte.

Hans Peter Peters

\section{Literatur}

Hayashi, Haruo (2006): The Role of Scientists in the Reporting of Natural Disasters. Vortrag bei der 9th International Conference on Public Communication of Science and Technology (PCST-9), Pre-Conference „Natural Disasters and Science Communication", Jeju, Südkorea, 14.-16. Mai 2006.

Schulz, Winfried (1976): Die Konstruktion von Realität in den Nachrichtenmedien. Analyse der aktuellen Berichterstattung. Freiburg, München.

\section{Mirko Marr}

\section{Internetzugang und politische Informiert- heit}

Zur digitalen Spaltung der Gesellschaft

Konstanz: UVK, 2005. - 255 S.

(Forschungsfeld Kommunikation; 19)

ISBN: 3-89669-475-8

Eine im Dezember 2005 erschienene Presseinformation zur Veröffentlichung der fünften Studie des „Digital Future Project“ (http://digitalcenter.org) der USC Annenberg School beginnt mit der Feststellung, dass „Internet users say that going online creates political clout" - eine Aussage übrigens, die vom folgenden Text nicht wirklich gestützt wird. Die Frage nach den Effekten des Internets bewegt auch Mirko Marr. Es fragt nicht nach dem Erwerb politischer "Schlagkraft" durch das Internet, sondern etwas bescheidener nach dem Zusammenhang von Internetnutzung und politischer Informiertheit. Marr versteht seine Arbeit, die im Jahr 2004 als Dissertation an der Universität Zürich angenommen wurde, als einen kritischen Beitrag zur Forschung über die digitale Spaltung der Gesellschaft. Seinen Grundgedanken hatte er bereits 2004 in einem Aufsatz in dieser Zeitschrift entwickelt (Wer hat Angst vor der digitalen Spaltung? Zur Haltbarkeit des Bedrohungsszenarios. M\&K 52, S. 76-94).

Er argumentierte damals, dass die „digitale Spaltung“ erst dann eine gesellschaftlich relevante Bedrohung darstelle, wenn nachgewiesen werden kann, dass die Nichtnutzung des Internets zu einer tatsächlichen Benachteiligung führt. Oder in den Worten von Wilhelm (2002): „[The] more fundamental issue [is] [...] not whether gaps exist, but what differences they make" (zitiert nach Marr, 2005: 228). Mit guten Argumenten und einer erfrischenden Polemik problematisiert er an den politischen Maßnah- 